\title{
Was genau gilt es zu bewältigen?
}

\section{Bewältigungsforschung, Rehabilitationspsychologie und Disability Studies ${ }^{1}$}

\section{Birgit Behrisch}

Journal für Psychologie, 26(2), 10-28

https://doi.org/10.30820/8248.02

www.journal-fuer-psychologie.de

\section{Zusammenfassung}

Behinderung rückt in der Rehabilitationspsychologie hauptsächlich als Thema von Bewältigung des Eintritts einer Beeinträchtigung oder chronischen Krankheit in den Blick. Dass dies die Perspektive auf Behinderung negativ einengt und eine Kultur behinderter Menschen von Affirmation und Selbstvertretung ausblendet, kritisieren die Disability Studies. Im Folgenden wird die sich daraus ergebende Debatte um das Konzept disability identity diskutiert. Unter Darstellung der Diskussionen um eine sociology of impairment wird umrissen, dass das ungeklärte Verhältnis von Beeinträchtigung und Behinderung als verschränkter Wechselwirkung zwischen Körper und Sozialität auch für den Ansatz psychology of disability innerhalb der Disability Studies eine offene Frage darstellt.

Schlüsselwörter: Rehabilitationspsychologie, Bewältigung, disability identity, psychology of disability, sociology of impairment

\section{Summary}

Coping with What? Coping, Rehabilitation Psychology and Disability Research In rehabilitation psychology disability appears primarily as a subject of coping with the onset of impairment or chronic illness. Disability Studies criticize that this restricts the perspective negatively and neglects a culture of disabled people including affirmation, self-representation and minority culture. In the following the debate around disability identity is discussed and, under representation of the discussions outlined around sociology of impairment, it is carved out that the unsettled relation between disability and impairment, as an entangled interaction between body and sociality, is also an open question for a psychology of disability.

Keywords: rehabilitation psychology, coping, disability identity, psychology of disability, sociology of impairment 


\section{Einleitung}

Die Themen Krankheit, Beeinträchtigung und Behinderung werden derzeit vor allem im Rahmen multiperspektivischer Zugänge diskutiert, die das Ergebnis eines langjährigen, kritischen Austauschs zwischen Medizin, Gesundheits- und Rehabilitationswissenschaften und den Disability Studies sind. Für die Disability Studies steht dabei die kontrovers diskutierte Perspektive des sozialen Modells im Mittelpunkt. Die Ebene der klinisch zu erfassenden Beeinträchtigung (impairment) wird im sozialen Modell von einer Ebene systematischer sozialer Unterdrückung und Ausschließung (disability) getrennt und letztere zu dem für die Disability Studies entscheidenden Analyse- und Aushandlungsgegenstand erhoben. Aus diesem Modell sind weitere Modellierungen hervorgegangen, die die Aspekte möglicher Affirmation eines Lebens mit Beeinträchtigung in einer kulturellen Gemeinschaft als einer gesellschaftlichen Minderheit betonen, im Gegensatz zu Ansichten, die Behinderung als eine persönliche Tragödie interpretieren. Dieses affirmative Modell von Behinderung findet mittlerweile im Ansatz psychology of disability innerhalb der Disability Studies Eingang in theoretische und empirische Arbeiten, vornehmlich der US-amerikanischen Rehabilitationspsychologie. Damit wird dezidiert von der üblichen Herangehensweise Abstand genommen, die Behinderung fast ausschließlich unter dem Gesichtspunkt der Krankheitsbewältigung bearbeitet.

Empirische Studien belegen, dass die Ausbildung eines affirmativen Selbstkonzeptes, beschrieben als disability identity, sich für behinderte Menschen als stabiler Faktor dafür erweist, mit den psychosozialen Belastungen aufgrund von gesellschaftlicher und sozialer Diskriminierung und Stigmatisierung bei einer Beeinträchtigung umzugehen (Bogart, 2014; Dunn \& Burcaw, 2013; Nario-Redmond et al., 2013). In der Rehabilitationspsychologie wird daher erörtert, inwieweit dies für (therapeutische) Angebote bezüglich der Identifikationsprozesse beeinträchtigter Menschen Anregung bieten kann. Bislang finden sich Selbstkonzepte einer disability identity nur bei einem, meist politisch aktiven oder interessierten, Teil der Menschen mit Behinderung (Putnam, 2005). Weiterhin wird diskutiert, wie die derzeitigen behindertenpolitischen Diskussionen die vornehmliche Selbstkategorisierung von Menschen mit Beeinträchtigung entlang einer bestimmten Diagnose hin zur Verwendung des Behinderungsbegriffs verändern werden.

Unter Berücksichtigung der Debatten um eine sociology of impairment soll im Folgenden aufgezeigt werden, dass auch für den Ansatz psychology of disability das in den Disability Studies umstrittene Verhältnis von Beeinträchtigung und Behinderung als verschränkte Wechselwirkung zwischen Körper und Sozialität eine offene Problematik darstellt. Zwar erweitert die Debatte um disability identity die hier aufgeworfene Frage, was genau es zu bewältigen gilt, um eine entscheidende affirmative 
Perspektive, sie steht aber in ihrer genaueren Ausarbeitung individueller Selbstkonzepte auf der Folie von Beeinträchtigung und Behinderung und im Zusammenhang mit sozialen Ordnungsprozessen und kollektiven Organisationsformen noch eher am Anfang.

\section{Behinderung als Thema der Rehabilitationspsychologie}

\subsection{Bewältigung als Thema von Krankheit und Behinderung}

Die psychologische Diskussion um Behinderung und chronische Krankheit erfolgt überwiegend in der Rehabilitationspsychologie. Damit ist deren Thematisierung gerahmt von den jeweiligen sozialpolitisch und rechtlich verfassten Systemen medizinischer und beruflicher Rehabilitation. Trotz der Vorsilbe $\gg$ Re- $\ll$, die aus der Tradition der Kriegsversehrtenförderung stammt (Brackhane, 1988), sieht die Rehabilitation ihre Aufgabenstellung nicht in einer alleinigen Wiedereingliederung, sondern zählt sowohl Menschen mit erworbener als auch angeborener Beeinträchtigung zur Zielgruppe und damit auch die Bereiche von schulischer Rehabilitation oder Frühförderung. Die spezifischen Zielsetzungen ergeben sich über das Behinderungsverständnis im jeweiligen Bereich; allgemein wird ein weitgefasster ganzheitlicher Ansatz zur Bearbeitung einer behindernden Wechselwirkung zwischen Individuum und Umwelt verfolgt. Ein psychologischer Schwerpunkt liegt dabei auf dem Thema Bewältigung, zumeist gefasst als Krankheitsbewältigung, was »alle Handlungen, Verhaltensweisen, emotionalen und kognitiven Prozesse [umfasst], die darauf abzielen, nicht nur mit der Krankheit, sondern auch mit dem Kranksein und seinen zahlreichen alltagsweltlichen, beruflichen, familiären und biographischen Implikationen umzugehen « (Wolf-Kühn \& Morfeld, 2016, S. 35).

Bewältigungsforschung fokussiert auf die Bearbeitung sogenannter kritischer Lebensereignisse, d.h. auf einschneidende, das Leben oft gravierend verändernde und in aller Regel außerordentlich belastende Erfahrungen, die das Person-Umwelt-Passungsgefüge zerstören (Filipp \& Aymanns, 2010). Die Bandbreite der Positionen reicht von einer stressorientierten Perspektive im Spannungsfeld von negativen bis ambivalenten (Gesundheits-)Folgen für das Individuum bis hin zu entwicklungsbezogenen Ansätzen mit passungstheoretischer Akzentuierung.

Charakteristika kritischer Lebensereignisse sind Schädigung der Person-UmweltPassung, Verluste, affektive Gewalt, mangelnde Kontrollierbarkeit, mangelnde Vorhersehbarkeit, Erschütterung des Weltbildes sowie des Selbstbildes und Selbstwertbezugs. Die Bewertung des Ereignisses als kritisch ist dabei ein relationaler Begriff und beschreibt ein Zusammenspiel der Merkmale der Situation und der Persönlichkeit des 
betroffenen Individuums (Filipp \& Ferring, 2002). Hildenbrand (2009) schlägt vor, Lebensereignisse hinsichtlich ihres normativen Gehalts zu unterscheiden. Das Ausbleiben normativer Lebensereignisse, wie z. B. Heirat oder Geburt eines Kindes, ist gesellschaftlich begründungspflichtig, was nicht für nicht-normative Ereignisse wie eine gesundheitliche Beeinträchtigung oder Behinderung gilt.

Bewältigungshandeln stellt keine homogene Klasse von Verhaltensweisen dar, sondern ist als ein relationales Geschehen in Bezug auf mentale und sozial-interaktive Aspekte, personale Ressourcen und Risiken anzusehen, wobei die Komponente Zeit eine entscheidende Rolle spielt. Hinsichtlich Zielsetzung und Bewertung von guter oder erfolgreicher Bewältigung rekurriert die Bewältigungsforschung auf $\gg$ wertnormative Prämissen« (Filipp \& Aymanns, 2010, S. 130). Kritisch anzumerken ist, dass kaum hinreichend geklärt ist, was eigentlich genau Bewältigung ist, zumal Aspekte sozialer Kontrolle, Normen und Regeln selten berücksichtigt werden (Knoll et al., 2017). In der Rehabilitationspsychologie wird Krankheitsbewältigung vor allem in Bezug auf chronische Erkrankungen theoretisiert. In einer stresstheoretischen Fassung meint Krankheitsbewältigung die Art und Weise, wie mit den Stressoren infolge körperlicher Beschwerden, reduzierter körperlicher Leistungsfähigkeit und dauerhaftem Fortbestehen der Erkrankung umgegangen wird. Konsequenzen auf psychischer, sozialer und Verhaltensebene werden zumeist negativ als Belastungsfolgen beschrieben, wobei auch persönliche Reifungsprozesse wie verstärkte Wertschätzung des Lebens, Erleben neuer Möglichkeiten, persönliche Stärke, religiöse und spirituelle Veränderung beschrieben werden (Krämer \& Bengel, 2016).

Neben Stress-Coping-Modellen in der Tradition der kognitiv-transaktionalen Stresstheorie (Lazarus \& Folkman, 1984) werden auch psychologische Theorien zu Selbstregulation und Selbstmanagement für das Thema Krankheitsbewältigung herangezogen. Ausgehend von den Studien von Corbin und Strauss (2004) wird Bewältigung als aktive Arbeitsleistung und Umsetzung individueller Handlungspläne im Rahmen biografischer, sozialer und krankheitsverlaufskurvenförmiger Prozesse und Ressourcen verstanden. Einen dritten maßgeblichen Theoriestrang bilden psychoanalytische Konzepte mit Blick auf Abwehrmechanismen und Ängste (Wolf-Kühn \& Morfeld, 2016). Im Hinblick auf Behinderung werden darüber hinaus verstärkt behavioristische, soziologische und körperschematheoretische Ansätze einbezogen (Budde, 1988; Lude \& Strubreither, 2015).

Als Einflussfaktoren auf die Bewältigung gelten Art, Schwere und Verlauf der Erkrankung und deren Bedeutung für die betroffene Person in ihrer individuellen Lebenssituation, welche geprägt ist vom sozioökonomischen Status sowie personalen, sozialen und gesellschaftlichen Ressourcen. Zu diesen zählen soziale Unterstützung, Zugang zu hilfreichen therapeutischen Angeboten, inklusionsförderliche Lebenswelten und materielle Sicherheit (Wolf-Kühn \& Morfeld, 2016). 


\subsection{Krankheit und Behinderung in bio-psychosozialer Perspektive}

Bewältigung in der Rehabilitationspsychologie meint in der Regel Krankheitsbewältigung, welche zumeist auch auf eine bestimmte Symptomatik fokussiert. Die Zielsetzung richtet sich auf die (Wieder-)Erlangung von Lebensqualität und funktionaler Gesundheit, was auch mit den veränderten konzeptionellen Beschreibungen von Krankheit und Gesundheit in den verschiedenen Disziplinen in den letzten Jahren zusammenhängt. So ist in den Gesundheitswissenschaften ein biomedizinisches Modell von einer biopsychosozialen Perspektive abgelöst worden, das neben krankheitsbedingter Symptomatik psychosoziale Aspekte von Belastungen und Ressourcen mittels Theorien zu Salutogenese und Resilienz untersucht. Auch der Begriff Behinderung ist durch die Weltgesundheitsorganisation in der International Classification of Functioning, Disability and Health (ICF) seit 2001 als bio-psychosoziales Modell gefasst. In der Rehabilitation ist dieses Konzept theorie- und handlungsleitend (Wolf-Kühn \& Morfeld, 2016). Behinderung wird hier als Teilhabeproblematik einer Wechselwirkung zwischen Person und Kontexten erfasst. Die Klassifikation erfolgt in Bezug auf Funktionsfähigkeit/funktionelle Gesundheit durch die Erfassung der Leistung und Leistungsfähigkeit einer Person. Weniger geht es damit um Krankheitsfolgen als um eine ressourcenorientierte Sichtweise bezüglich verschiedener Komponenten von Gesundheit, womit in der Zielsetzung » gegen das in der kurativen Medizin angestrebte Ziel der Heilung und für eine der Realität in der Rehabilitation angepassten Zielfindung « (Mäder, 2015, S. 16) optiert wird. Wichtige rehabilitationspsychologische Themen von Diagnostik und Intervention liegen in den Bereichen Krankheitsmanagement und Behinderungsverarbeitung, in der Ermittlung von Ressourcen und sozialer Unterstützung und der Stärkung gesundheitsfördernden Verhaltens mittels Patientenschulungen.

Das Modell der ICF wird dabei als Kompromiss angesehen zwischen einem medizinischen, eher personenzentrierten, defizitorientierten Modell einerseits und einem sozialen Ansatz andererseits, der Behinderung in sozialen Interaktionsprozessen behinderter Menschen mit ihrer Umwelt verortet (Wolf-Kühn \& Morfeld, 2016). Allgemein etabliert die ICF in ihrer theoretischen Modellierung ein breites Verständnis von Behinderung (einschließlich chronischer Erkrankungen) unter Berücksichtigung von Teilhabe und Kontextfaktoren. Als handlungspraktisches Instrumentarium findet die ICF momentan allerdings eher wenig konsequente Anwendung. So besteht für die Zielsetzung einer globalen einheitlichen Begriffsfassung durch die ICF derzeit die »Gefahr einer ausufernden unsystematischen Nutzung des biopsychosozialen Modells « (Wenzel \& Morfeld, 2017, S. 391). Dies liegt vor allem an der ungleichen Gewichtung der Komponenten des bio-psychosozialen Ansatzes. In dessen Operationalisierung sind die sozialen Komponenten schwach vertreten bzw. können im Hinblick auf Aktivitäten und Partizipation als unterbestimmt bezeichnet werden (Oliver \& Barnes, 2012). Für 
die personenbezogenen Faktoren im Bereich spezifischer subjektiver Wahrnehmungen und Bewertungen sowie Bewältigungsstrategien liegen bislang, auch aufgrund ethischer Bedenken, keine offiziellen Parameter vor (Lude \& Geyh, 2015). Hinzu kommt die normative Orientierung an einem Leistungs(fähigkeits)begriff, der Beeinträchtigung als negative Abweichung von Körpernormen konzeptualisiert, ohne dass kulturelle und kontextuelle Einflüsse solcher Einschätzungen thematisiert werden. Zusammenfassend bewerten Oliver und Barnes aus der Perspektive des britischen sozialen Modells von Behinderung die ICF als einen ideologischen Ausdruck von $\gg$ methodological individualism « und »investigative foundationalism « (Oliver \& Barnes, 2012, S. 27). Auch Hirschberg bescheinigt der ICF in einer diskursanalytischen Betrachtung ein janusköpfiges Gesicht:

»Die Forderungen zielen darauf ab, die Konzeption der ICF in Richtung auf Teilhabe und Selbstbestimmung behinderter Menschen zu verbessern. Die Befürchtungen beziehen sich hingegen auf ein verändertes Verständnis von Behinderung und Funktionsfähigkeit, in dem der Körper jedes Subjekts als optimierungswürdig beurteilt wird « (Hirschberg, 2009, S. 326).

\section{Einwürfe einer psychology of disability}

\subsection{Entdramatisierung und Affirmation}

Seitens der Disability Studies werden rehabilitationswissenschaftliche Ansätze zu Behinderung dahingehend kritisiert, dass sie Behinderung eher negativ und einseitig bestimmen (Goodley, 2013; Olkin, 2003; Reeve, 2006). Behinderung thematisiert die Rehabilitationspsychologie zumeist als erworbene Beeinträchtigung, die die jeweils betroffene Person und ihr unmittelbares Umfeld vor die Notwendigkeit stellt, sämtliche Lebensbereiche - von persönlichen Beziehung über Wohnen bis Arbeit - neugestalten zu müssen. Aus diesem Ansatz resultiert eine Konzeptionierung von Behinderung als Psychopathologie, die verbunden ist mit den Themen Bewältigung, Verlust und Trauer (Reeve, 2006). Erkenntnisse resultieren dabei mehrheitlich aus dem Prozess der Anpassung vormals nicht behinderter Menschen an ihre Beeinträchtigung. Zugrunde liegt dem die Vorstellung von Behinderung als einer persönlichen Tragödie. Zudem führt die Begrenzung auf die Subdisziplin der Rehabilitationspsychologie dazu, dass Behinderung nicht als ein Querschnittsthema der Psychologie etabliert wird.

Das personal tragedy model wird von French und Swain (2004) genauer gefasst. Die Ansicht, Behinderung sei ausschließlich in Konzepten von Leiden und Verderben zu denken, entsteht auf der Grundlage wirkmächtiger Annahmen nicht behinderter 
Menschen über das Leben behinderter Menschen. Nicht behinderte Menschen bringen das Thema Behinderung gemäß den sozialen Wertmaßstäben mit Abhängigkeit und Abnormität in Verbindung. Behinderung wird als Tragödie interpretiert, auf welche ausschließlich mit Heilung und Normalisierung reagiert werden sollte. Aus kulturwissenschaftlicher Perspektive resümiert Cameron: »The personal tragedy model is the cultural expression of the individual or medical model and is materialised through the recycling of disciplinary messages that >ablebodieness < is valued while impairment is a mark of misfortune « (Cameron, 2014b, S. 117).

Goodley (2013) stellt diesem Verständnis der Disziplin einer psychology of impairment seinen Ansatz einer psychology of dis/ability and disablism entgegen. Schwerpunkt dabei ist ein allgemein-psychologischer Blick auf Behinderung im Sinne des sozialen Modells nach Thomas (1999), das die Erfahrungen sozialer Marginalisierung behinderter Menschen reflektiert und die Effekte behindernder Verhaltensweisen und Umwelten auf ihr emotionales Wohlbefinden miteinbezieht. Bereiche des psychosozialen Disablismus betreffen Erfahrungen der Exklusion aufgrund von Barrieren sowie die schwierige Interaktions- und Beziehungsgestaltung zwischen Menschen mit und ohne Behinderung aufgrund von Vorurteilen oder Uninformiertheit. Anhand des Modells lässt sich auch die Internalisierung von abwertenden Urteilen und deren Übernahme in das Selbstwertkonzept thematisieren (Reeve, 2006).

French und Swain (2004) verweisen darauf, dass mittels einer psychosozialen Perspektive auch ein kulturelles Selbstverständnis behinderter Menschen formuliert werden kann, welches Identität in positiven statt negativen Kategorien fasst:

$>\quad \gg$ Being different and thinking differently about being different, both individually and collectively

$>$ The affirmation of unique ways of being situated in society

$>$ Disabled people challenging presumptions about themselves and their lives in terms of not only how they differ from what is average or normal, but also about the assertion, on their own terms, of human embodiment, lifestyles, quality of life and identity

$>$ Ways of being that embrace difference « (Swain \& French, 2008, S. 185, zit.n. Cameron, 2014a).

Im affirmation model geht es darum, die kulturell dominante Beschreibung herauszufordern, welche Beeinträchtigung allein als Verlust und jenseits der Normalität beschreiben kann, sowie darum, auf die Möglichkeit eines respektvollen Umgangs mit körperlicher Verfasstheit jeglicher Art hinzuweisen, auch im Zusammenhang mit Geschlecht, sexueller Orientierung, Alter etc. (Swain \& French, 2000). In Anlehnung an die Bestimmung von impairment und disability durch das soziale Modell (UPIAS, 1976) werden beide Begriffe unter Einbeziehung des kulturell-rahmenden Kontextes bestimmt. 


\begin{tabular}{|l|l|l|}
\hline & $\begin{array}{l}\text { Social model } \\
\text { (UPIAS, 1976) }\end{array}$ & $\begin{array}{l}\text { Affirmation model } \\
\text { (Cameron, 2014a) }\end{array}$ \\
\hline Impairment & $\begin{array}{l}\text { lacking part of or all of a limb, or } \\
\text { having a defective limb, organ or } \\
\text { mechanism of the body }\end{array}$ & $\begin{array}{l}\text { physical, sensory, emotional and } \\
\text { cognitive difference, divergent } \\
\text { from culturally valued norms of } \\
\text { embodiment, to be expected and } \\
\text { respected on its own terms in a } \\
\text { diverse society }\end{array}$ \\
\hline Disability & $\begin{array}{l}\text { the disadvantage or restriction of } \\
\text { activity caused by a contemporary } \\
\text { social organisation which takes } \\
\text { little or no account of people who } \\
\text { have physical impairments and } \\
\text { thus excludes them from the } \\
\text { mainstream of social activities }\end{array}$ & $\begin{array}{l}\text { a personal and social role which } \\
\text { simultaneously invalidates the } \\
\text { subject position of people with } \\
\text { impairment and validates the } \\
\text { subject position of those } \\
\text { considered normal }\end{array}$ \\
\hline
\end{tabular}

\subsection{Affirmative Ansätze und disability identity (development)}

Die Diskussion um Anerkennung und Affirmation einer kulturellen Szene behinderter Menschen wird im Rahmen der US-amerikanischen Disability Studies bereits länger geführt. Longmore (1995) sieht in den 1990er Jahren die Behindertenbewegung in eine zweite Phase eintreten, in der, auch in Orientierung an weitere Bürgerrechtsbewegungen, nach der Durchsetzung von Rechten behinderter Menschen eine Behindertenkultur von disability pride und culture movement etabliert wird. In den letzten Jahren findet diese Diskussion verstärkt Eingang in die englischsprachige (Rehabilitations-)Psychologie. Seitens der Positiven Psychologie beginnt eine dezidierte Auseinandersetzung mit Behinderung und der Ausprägung verschiedener behinderungsbezogener Identitäten mittels einer an Stärken orientierten Herangehensweise mit einem Fokus auf Themen wie Hoffnung, Optimismus und Lösungsorientierung (Wehmeyer, 2013). Im therapeutischen Bereich existiert schon länger der Ansatz der disability-affirmative therapy, die sich im Sinne sozialer Gerechtigkeit als Arbeit mit Angehörigen einer diskriminierten Minderheitengruppe versteht (Olkin, 2012). Ausgangspunkt sind social model und minority model, welche von therapeutischer Seite aus die Interventionen strukturieren und auf der Seite der Klient*innen verschiedene Sichtweisen von Behinderung für das eigene Selbstbild wertschätzend zulassen - beispielsweise auch eine moralische oder religiöse Interpretation von Behinderung als Manifestation von Sünden oder als Test der Stärke. Disability-affirmative therapy wird als cross-culture therapy definiert, welche sich die Situation zwischen nicht behinderten 
Therapeut*innen und behinderten Patient*innen in ihren (Macht-)Effekten verdeutlichen muss. Seitens der Therapeut*innen bedarf es einer gewissen Kenntnis über Behinderungsmodelle sowie kulturelle und politische Aktivitäten der Behindertenbewegung, da diese zumeist den Zugang zu Kultur und Gemeinschaft behinderter Menschen gestalten. Die Teilnahme an solchen Aktivitäten sollte Patient*innen weder aufgedrängt noch sollte der Wunsch danach ignoriert werden (Dunn \& Burcaw, 2013).

Einen Schwerpunkt in der Debatte um affirmative Ansätze in der Rehabilitationspsychologie bilden Modelle einer disability identity, verstanden als soziale Identität, als Mitglied einer stigmatisierten Minderheitengruppe. Hierbei geht es weniger um die in der Soziologie geführte Diskussion um Identitätsentwicklung und behinderte Identität im Zusammenhang mit Stigma-Management, Diskriminierungserfahrungen und der Verletzung persönlicher Grenzen, die in der deutschen Literatur bereits (kritisch) besprochen werden (Mürner \& Sierck, 2011). Vielmehr geht es um eine Auslegung des sozialen Modells als minority group model of disability, welches die Bedeutung öffentlicher Politik für die Änderung gesellschaftlicher Diskriminierung, Stigmatisierung und Unterdrückung der Gruppe behinderter Menschen als disabled minority postuliert:

$>\quad \gg$ That the source of the major problems confronting disabled people can be attributed primarily to social attitudes;

$>$ that almost every facet of the environment has been shaped or molded by public policy; and

$>$ that, at least in a democratic society, policies are a reflection of pervasive attitudes and values « (Hahn, 1994, S. 4, zit.n. Putnam, 2005).

Ein starker theoretischer Bezugspunkt des minority group model of disability ist die Theorie der sozialen Identität und die davon abgeleitete Selbstkategorisierungstheorie (Tajfel \& Turner, 1979). Die soziale Identität eines Individuums bildet sich über die Mitgliedschaft in einer sozialen Gruppe (Eigengruppen) und den damit in Zusammenhang stehenden kognitiven und emotionalen Wertigkeiten. Menschen sind bemüht, ein positives Selbstkonzept zu erreichen, unter anderem auch über die Abwertung von Fremdgruppen. In Anlehnung daran wird mit dem Begriff disability identity nach der Entwicklung positiver Selbstkonzepte in Bezug auf die Identifikation mit der sozialen Gruppe behinderter Menschen gefragt. Die Literatur in diesem Bereich ist im Gegensatz zu den Themen sexuelle und kulturelle Identität recht überschaubar: In einer aktuellen Literaturrecherche in der Datenbank PsycInfo finden sich 52 Einträge (Forber-Pratt \& Zape, 2017) und lediglich in wenigen Publikationen ist eine genauere theoretische Auseinandersetzung zu finden (Forber-Pratt et al., 2017). Zur genaueren Bestimmung der Thematik wird im Folgenden die Entwicklung der Debatte in ihren theoretischen und methodischen Bezügen dargestellt. 
Gill (1997) rechnet sich selbst der Behindertenbewegung in den USA zu und entwickelt vier Typen der Integration auf der Grundlage von Gesprächen mit Aktivist*innen und Klient*innen (siehe auch Forber-Pratt et al., 2017). Mit Bezug auf Longmore (1995) geht es um die Klärung individueller Identität im Zusammenhang mit sozialer Gruppenidentität als Angehörige einer Minderheit. Die vier Typen umfassen (vgl. Gill, 1997, S. 42-45):
$>$ coming to feel we belong (integrating into society),
$>$ coming home (integrating with the disability community),
$>$ coming together (internally integrating our sameness and differentness),
$>$ coming out (integrating how we feel with how we present ourselves).

In Anlehnung an Beschreibungen von Identitätsprozessen weiterer Minderheiten beschreibt Gill (1997) verschiedene Adaptionsprozesse - von Assimilationsversuchen an die Mehrheitsgesellschaft über eine Phase der Separation hin zu einer persönlichen Identität, die auch den Stolz auf die eigene Bezugsgruppe einschließt:

$\gg$ The $>$ coming out< process is often the last step toward disability identity in a path that begins with a desire to find a place in society, continues with a discovery of one's place in a community of peers, and builds to an appreciation and acceptance of one's whole self complete with disability« (ebd., S. 45).

Das Spannungsverhältnis von Behinderung und Heilung untersuchten Hahn und Belt (2004) mittels einer größeren Fragebogenstudie unter Aktivist*innen der ADAPT (Americans Disabled for Accessible Public Transport). Auf der theoretischen Folie der disabled minority wurde ein Fragebogen in Anlehnung an Skalen zur Erfassung von ethnic identity development konstruiert. Gefragt wurde nach der Art der Verbindung zur Gruppe von Menschen mit Behinderung (communal attachments), ob eine positive Identifikation als behinderter Mensch (personal identity) vorliegt und ob eine mögliche Ablehnung von Heilung auch im Falle der Existenz einer Wunderpille beibehalten werden würde. In Bezug zum Thema Heilung konnten die Autoren die entscheidende Rolle eines affirmativen Selbstkonzepts herausarbeiten: »Individuals with stronger positive affirmation of personal identity as being disabled are more likely to reject a cure because curing their disability takes away their source of self affirmation « (Hahn \& Belt, 2004, S. 460).

Der Befund, dass politischer Aktivismus auf der Interaktion von Gruppen und Organisationen beruht, welche Informationen teilen und Peer-Beziehungen aufbauen, veranlasste Putnam (2005) zu der Frage, wie eine derartige gruppenbezogene Identität internalisiert wird. Mittels Literaturanalyse arbeitet sie sechs Primärparameter heraus, die eine political disability identity strukturieren. Sie verweist darauf, dass nur ein Teil der behinderten Menschen eine Selbstpositionierung als Aktivist*in vornimmt: 
$>$ Self-Worth - Überzeugung, dass Menschen mit Behinderung und Menschen ohne Behinderung gleichwertig sind und einen produktiven Beitrag zur Gesellschaft leisten können.

$>$ Pride - Überzeugung, dass Behinderung ein Aspekt menschlicher Vielfalt ist, wobei die Beeinträchtigung nichts inhärent Negatives ist, dies aber durch kulturelle, soziale oder bauliche Faktoren werden kann.

$>$ Discrimination - Überzeugung, dass Menschen mit Behinderung negativ stereotypisiert werden und Diskriminierung ein Ergebnis von Chancenungleichheit und des ungleichen Zugangs zu sozialen und ökonomischen Ressourcen ist.

$>$ Common Cause - Überzeugung, dass Menschen mit Behinderung Ähnliches erleben und sich einige dieser Erfahrungen ändern müssen, wozu es einer gemeinsamen politischen Agenda bedarf.

$>\quad$ Policy Alternatives - Überzeugung, dass Behinderung nicht im Individuum liegt und Möglichkeiten, behindernde Erfahrungen abzubauen, mittels politischer Entscheidungen erreicht werden können.

$>$ Engagement in Political Action - Überzeugung, dass behinderte Menschen eine eigene politische Vertretung stellen sollten, welche eine Minderheit darstellt, aber mittels Engagement auf politische Entscheidungen Einfluss nehmen kann.

Auf der Basis des sozialen Modells in seiner Auslegung durch Hahn und Belt (2004) sowie des affirmativen Modells von Swain und French (2000) entwickelt Darling (2003) das concept of disability orientation. Dieses erfasst neben disability identity auch das Niveau des politischen Engagements einer Person. Aus den Vorarbeiten entstand der Fragebogen Questionnaire on Disability Identity and Opportunity (QDIO) (Darling \& Heckert, 2010), der in einer größeren Studie mit Proband*innen ab dem Jugendalter mit verschiedenen Beeinträchtigungen genutzt wurde. Die Ergebnisse streuen breit hinsichtlich der Orientierungen, die in den Kategorien von Disability Pride, Exclusion/Dissatisfaction, Social Model sowie Personal/Medical Model angesprochen werden.

Auch Forber-Pratt und Zape (2017) fragen, ob die Diskussionen über ein verändertes Behinderungsbild mittlerweile in Identitätskonzepten behinderter Menschen ihren Niederschlag finden. In einer qualitativen Studie auf der Grundlage von Interviews und Beobachtungen werden Studierende als Vertreter*innen der »ADA-Generation « d.h. der Generation, für welche der American with Disability Act (ADA) eine Selbstverständlichkeit darstellt - in den Fokus gerückt. Aus ihrer Untersuchung leiten die Autor*innen ein Model of Social \& Psychosocial Disability Identity Development ab. Beziehungen, Mitwirkung und Engagement in der Bezugsgruppe behinderter Menschen werden als wichtige Aspekte einer disability identity angesehen und in vier Status beschrieben (wobei sich Personen gleichzeitig in verschiedenen Status befinden können): $>$ Acceptance-Man selbst und wichtige Bezugspersonen akzeptieren die Behinderung. 
$>$ Relationship - Man trifft andere Menschen wie man selbst und engagiert sich in deren Verständnis.

$>$ Adoption - Man teilt die Werte der Gruppe.

$>$ Engagement - Man unterstützt andere Gruppenmitglieder und wird vielleicht selbst zum Rollenvorbild.

\subsection{Zwischen Selbstidentifikation und Ablehnung}

Die Ausprägung einer disability identity im beschriebenen Sinne von Affirmation und Gruppenzugehörigkeit erweist sich als Prädiktor für höhere Werte von Lebenszufriedenheit sowie Selbstwirksamkeit und schützt das Wohlbefinden (Bogart, 2014; Dunn \& Burcaw, 2013; Nario-Redmond et al., 2013). Der Anschluss an die Selbstvertretung erzielt zudem positive Sekundäreffekte auf der Ebene sozialer Unterstützung und gesundheitlicher Beratung und Austausch unter Betroffenen (Forber-Pratt \& Zape, 2017). Gerade in Bezug auf die positive Verarbeitung psycho-emotionaler Belastung im Zusammenhang mit behindernden Erfahrungen von Diskriminierung und Fremdheit scheint diese Identitätsausprägung ein Vorteil zu sein gegenüber einer Identifikation, welche allein auf die spezifische Beeinträchtigung fokussiert, was Bogart et al. (2017) als Paradox werten:

»This may seem ironic that those who are most sensitive to recognizing prejudice or to attributing negative outcomes to ableism are the same people who derive the most protection from internalized oppression due to stigma. The group protects the individual and individuals are more apt to work on behalf of the group when the group is claimed as part of self-definition « (Nario-Redmond \& Oleson, 2016, S. 216).

Inwieweit die Debatte um disability identity Eingang finden wird in Prozesse von Rehabilitation und Beratung, ist derzeit nicht abzusehen. Während andere soziale Identifikationen positive Ausprägungen erfahren haben - etwa black pride oder gay pride wird disability pride oftmals als Oxymoron verstanden (Bogart et al., 2017). Zwar finden sich Forderungen nach einer Anpassung von rehabilitativen und politischen Strategien zur Förderung von Gemeinschaftsstrukturen sowie von positiven Identifikationsmöglichkeiten, aber die Debatte in der Psychologie steht erst am Anfang. Der Terminus disability identity, der sich auf eine positiv konnotierte Selbstdefinition bezieht, erfasst lediglich das Selbstkonzept eines Teils der Gruppe behinderter Menschen. Zum einen beschreiben nicht alle Menschen, die als behindert betrachtet werden, sich selbst als behindert. Zum Beispiel bezeichnen sich zwei Drittel der Menschen mit körperlicher Beeinträchtigung als behindert, nimmt man allerdings nur die Gruppe älterer Menschen, lehnen zwei Drittel diese Eigenbezeichnung ab (Darling \& Heckert, 2010). 
Zum anderen erfolgt eine Selbstbeschreibung als behindert nicht nur in positiven, sondern auch in negativen oder ambivalenten Bezügen. Eine Vielzahl behinderter Menschen kategorisiert sich mittels einer Diagnose, was dem nach wie vor wirkmächtigen medizinischen Modell zugeschrieben wird, aber auch dem Umstand, dass behinderte Menschen oft allein unter nicht behinderten Menschen leben (Bogart et al., 2017).

Studien, welche ihre jeweiligen Modelle von disability identity empirisch über Fragebögen oder Interviews erarbeitet haben, verweisen auf die zwei Kernindikatoren angeboren/erworben und Sichtbarkeit/Unsichtbarkeit, die entscheidend eine Entwicklung positiver Selbstidentifikation als behindert ermöglichen oder verhindern (Bogart, 2014; Darling \& Heckert, 2010; Hahn \& Belt, 2004; Nario-Redmond et al., 2013). In einer vergleichenden Studie zwischen den Gruppen von Menschen mit erworbenen und angeborenen Behinderungen zeigen Menschen mit angeborener Behinderung eine höhere Lebenszufriedenheit, wobei ein früher biografischer Erwerb in Kindheit und Jugend entscheidend ist, nicht jedoch der Umstand, wie lange eine Person bereits mit der Behinderung lebt (Bogart, 2014). Hahn und Belt (2004) vermuten, dass bei einem späten Eintritt einer Beeinträchtigung sozialisierte (abwertende) Normen gegenüber Behinderung die Ausbildung von disability pride verhindern. Dies wirft die Frage auf, ob es eine »critical period for optimal adaptation to disability « (Bogart, 2014, S. 113) gibt, die möglicherweise in der Kindheit und vor Abschluss wichtiger Entwicklungsschritte liegt. Hinsichtlich der Sichtbarkeit der Beeinträchtigung wird diskutiert, warum sich eine disability identity eher bei Personen ausbildet, die ihre Behinderung nicht verbergen können und damit vermehrter Erfahrung von Stigmatisierung ausgesetzt sind. Zudem können diese Personen ihre Beeinträchtigung nicht geheim halten und in den Augen anderer zur Gruppe nicht behinderter Menschen gehören.

\section{Schnittpunkte zwischen sociology of impairment und psychology of disability}

In den letzten Jahren haben sich die Debatten in der Rehabilitationspsychologie, den Gesundheitswissenschaften und den Disability Studies gegenseitig beeinflusst und Aspekte von Krankheit, Beeinträchtigung und Behinderung in einem bio-psychosozialen Rahmen verortet. Besonders die Perspektive chronischer Erkrankungen konnte damit enger mit sozialen Aspekten von Behinderung verknüpft werden (Zander, 2015). Dieser interdisziplinäre Dialog ist aber auch von einer gewissen begrifflichen Unbestimmtheit geprägt, etwa in Bezug auf die Termini Teilhabe oder Inklusion. Besonders kritische (politische) Aspekte des sozialen Modells der Disability Studies geraten damit teilweise in den Hintergrund. Es ist grundsätzlich zu fragen, ob ein Instrument funktionaler Gesundheit wie die ICF soziale Kriterien erfassen und operationalisieren kann, welche sich 
aus komplexen historisch und kulturell gebundenen Aushandlungsprozessen für bestimmte (wohlfahrts-)staatliche, gesellschaftliche Konstellationen ergeben. Mit Oliver und Barnes (2012) ist darauf hinzuweisen, dass Behinderung vorrangig ein (sozial-)politisches Phänomen ist und weniger ein Gegenstand der Fürsorge und der Medizin.

Der Ansatz der psychology of disability kritisiert an der Rehabilitationspsychologie, dass die Theoretisierung von Bewältigung eher am Phänomen Beeinträchtigung als an Behinderung erfolgt, weil vorrangig die Personengruppe mit erworbener Behinderung in den Blick genommen wird. Generell ist für die Theoretisierung von Beeinträchtigung und Behinderung kritisch nachzufragen, mit welcher Personengruppe genau argumentiert wird. In empirischen Studien zum affirmativen Modell einer disability identity zeigt sich, dass die zwei Indikatoren angeborene/erworbene Behinderung und Sichtbarkeit/Unsichtbarkeit der Behinderung ausschlaggebend dafür sind, ob die Entwicklung einer positiven Selbstidentifikation als behindert möglich wird. Beide Indikatoren verweisen auf theoretischer Ebene auf die Körpergebundenheit der Phänomene Beeinträchtigung und Behinderung, was in der Debatte um disability identity selbst nicht weiter thematisiert wird. Zwar erfolgt die Argumentation auf der Basis des social model von Behinderung, die Kritik der Körpervergessenheit gegenüber dem sozialen Modell wird allerdings nicht weiter problematisiert.

Der Einwurf, welcher die Ausklammerung des Körpers aus der Konzeption von Behinderung betrifft, erfolgt aus unterschiedlichen Theoriesträngen. In einer kulturwissenschaftlichen Herangehensweise wird die Bedeutung historisch und kulturell gebundener Körpernormen und Normalitätsvorstellungen für die (De-)Konstruktion von Behinderung herausgearbeitet (Waldschmidt, 2005). Eine Art Leibvergessenheit des sozialen Modells, d.h. der fehlende Einbezug persönlicher, durchaus auch negativer Erfahrungen von Beeinträchtigung und der daraus erwachsenen Expertise über den eigenen Körper, wird vorrangig aus phänomenologischer Sicht thematisiert (Hughes \& Paterson, 1997; Wendell, 1999) und hat zur Forderung nach einer eigenständigen sociology of impairment geführt. Während dabei zu Beginn Aspekte der narrativen Abbildung persönlicher Wahrnehmung im Fokus standen, werden momentan im Anschluss an Theorien sozialer Ungleichheit und Intersektionalität Fragen struktureller Bedingungen von Beeinträchtigung thematisiert:

»The cultural construction of impairment is not the same as the cultural construction of disability. They obviously overlap, but they are not the same. Not all cultural constructions of impairment are disabling. Similarly, not all bodies or impairments have the same meaning in the cultural construction of citizenship rights or the nation more broadly « (Sherry, 2016, S. 734).

Neuere Fragestellungen richten sich auf kulturelle und zeitliche Rahmungen von Diagnosen und Medikalisierung und darauf, inwieweit diese Phänomene mit weiteren 
Kategorisierungen wie Gender, sexueller Orientierung, Ethnizität und sozioökonomischen Aspekten zusammenhängen und welche Rolle Diagnosen im Rahmen von Ressourcenallokation und Selbstvertretung sowie für Bildung und Sozialpolitik spielen.

Für die Disability Studies bringt die Zweiteilung von Beeinträchtigung und Behinderung den analytischen Gewinn, soziale Aspekte von Diskriminierung und Exklusion jenseits der Debatte um körperliche Funktionsfähigkeit zu thematisieren. Allerdings führt Tremain (2002) kritisch an, dass weder behinderte noch nicht behinderte Menschen einer Eigendefinition von behindert z. B. aufgrund der Hautfarbe zustimmen würden, weshalb sie die Trennung von Behinderung und Beeinträchtigung im sozialen Modell als Chimäre bezeichnet. Im Ganzen ist diese Trennung von Körper und Sozialem nicht ganz unproblematisch, worauf mittels körpersoziologischer Ansätze hingewiesen werden soll. Sozialität ist als » verkörperte Sozialität « (Lindemann, 2005, S. 125) zu begreifen, in welcher der Körper kein Erkenntnisanhängsel ist und in seiner Dualität als andauernder gegenwärtiger Leib erst die Möglichkeit eines umweltlichen Zugangs überhaupt bedingt. Dabei ist der Körper als Produkt von Gesellschaft in Aspekten von Formung, Diskurs, Umwelt und Leib anzusehen wie auch als Produzent in Routinen und Inszenierungen, aber auch im körperlichen Eigensinn (Gugutzer \& Schneider, 2007). Die Formel »disability is embodied and impairment is social « (Hughes \& Paterson, 1997, S. 336) ist dahingehend zu schärfen, dass abgebildet wird, wie Körper in soziale Dynamiken eingebunden sind und diese im Gegenzug bis auf die Ebene des Leibes von sozialen Effekten bestimmt werden (Behrisch, 2014; Connell, 2011). Erst darüber werden Widersprüche der Erfahrungen von Menschen mit angeborenen und erworbenen Beeinträchtigungen sowie unterschiedliche Einstellungen zu Fragen von Heilung, Technikgebrauch und politischem Engagement bearbeitbar werden.

Weiterhin ist in der Betrachtung sozialer Dynamiken die sozialrechtliche und politische Ebene entscheidend. Das Phänomen Behinderung, so wie es sich in westlichen Ländern sozialpolitisch konstituiert, ist an Beeinträchtigung gebunden. Daher werden Aspekte der Lebenslage, die mit Beeinträchtigung und Behinderung im Zusammenhang stehen, gesellschaftlich vor allem in den Bereichen Gesundheit und Soziales organisiert und verhandelt: Medizinisch-therapeutische Angelegenheiten von Intervention, Medikation, Hilfsmittel, Pflege und Gesundheitsstrategien werden auf der Basis einer bestimmten Diagnose erörtert. Ansprüche auf Nachteilsausgleiche, Rehabilitation und Eingliederungshilfen werden sozialrechtlich um den Terminus Behinderung organisiert. Für beide Bereiche bedarf es einer ungeheuren Bandbreite an Kenntnissen oder auch literacy (Sherry, 2016). Dies betrifft nicht allein das Wissen, dass behinderte Menschen über die ihnen zustehenden Rechte haben sollten, sondern auch Kenntnisse hinsichtlich der rechtlichen und administrativen Ermöglichungs- und Durchsetzungsmöglichkeiten (Behrisch, 2014). Möglichkeiten von Selbstorganisation und Identifikation sind damit zum einen durch eine spezifische Diagnose und zum anderen durch den Begriff Behinderung gegeben. 
Aufgrund der Trennung zwischen Selbsthilfe und Selbstvertretung werden gesundheitsbezogene Selbsthilfe und auch Behindertenorganisationen entlang von Diagnosen innerhalb der Disability Studies kritisch besprochen. Bogart et al. (2017) sehen in der Identifikation über eine Diagnose ein Hindernis für die Ausbildung einer disability identity, weswegen der Weiterentwicklung und Stärkung der Behindertenbewegung ein besonderer Stellenwert zugesprochen wird. In den angesprochenen Studien werden die Rahmungen und Ressourcen für personale und soziale Identität in Bezug auf die Behindertengemeinschaft ausschließlich über den je persönlichen Blickwinkel beschrieben. Inwieweit die Behindertenbewegung eine kollektive Identität im Zusammenhang mit Hierarchisierungs-, Ein- und Ausschließungsprozessen unter anderem auf der Grundlage von Beeinträchtigungen hervorbringt, ist jedoch selten Thema (Deal, 2003). Hierin wird ein Hauptauftrag einer sociology of impairment gesehen:

»At a minimum, a sociology of impairment must explore such issues as the widespread personal refusal of impairment identification; expression of single-impairment identity politics; the significance of single-impairment organizations for collective organization and resource allocation; difficulties sustaining multiple-impairment and cross-impairment political organization; contradictions between organizations $>$ of $<$ and organizations $>$ for $<$ people with impairments; the creation of impairment/disability hierarchies; and the political challenges for those who wish to organize around multiple identities (Sherry, 2016, S. 737).

Darling beschreibt die Disability Studies als »a mix of empirical research and ideological writings « (Darling, 2003, S. 894) und verweist darauf, dass dabei der empirische Wissensstand im Hinblick auf Identitäts- und Rollenverständnisse behinderter Menschen derzeit als gering anzusehen ist. Generell, besonders für das Thema disability identity, ist eine breite Perspektive auf die Identifikation entlang Beeinträchtigung und Behinderung nötig, welche auf individueller und kollektiver Ebene Narrationen ebenso einbezieht wie (soziale und politische) Organisationsprozesse. Dies wäre ertragreich sowohl für die sozialwissenschaftlich orientierten Disability Studies, insbesondere die sociology of impairment, als auch für eine Rehabilitationspsychologie im Sinne einer psychology of disability.

\section{Anmerkung}

1 Im anglo-amerikanischen Sprachraum lässt sich eine vermehrte Bezugnahme der Rehabilitationspsychologie auf die Disability Studies beobachten. Ziel dieses Artikels ist es, diese Debatten für die Besprechungen im deutschsprachigen Raum auszuloten. Englische Bezeichnungen für Konzepte, Modelle und Diskursstränge, welche bislang kaum Eingang in deutschsprachige Debatten gehalten haben, werden daher (vorerst) beibehalten. 


\section{Literatur}

Behrisch, B. (2014). »Ein Stücknormale Beziehung«. Zum Alltag mit Körperbehinderung in Paarbeziehungen. Bielefeld: transcript.

Bogart, K. R. (2014). The role of disability self-concept in adaptation to congenital or acquired disability. Rehabilitation Psychology, 59(1), 107-115.

Bogart, K.R., Rottenstein, A., Lund, E.M. \& Bouchard, L. (2017). Who self-identifies as disabled? An examination of impairment and contextual predictors. Rehabilitation Psychology, 62(4), $553-562$.

Brackhane, R. (1988). Behinderung, Rehabilitation, Rehabilitationspsychologie: Terminologische Vorbemerkungen und Begriffsklärungen. In U. Koch, G. Lucius-Hoene \& R. Stegie (Hrsg.), Handbuch der Rehabilitationspsychologie (S. 20-34). Berlin, Heidelberg: Springer.

Budde, H.-G. (1988). Auswirkungen und Bewältigung von Behinderung: Psychologische Ansätze. In U. Koch, G. Lucius-Hoene \& R. Stegie (Hrsg.), Handbuch der Rehabilitationspsychologie (S. 101-119). Berlin, Heidelberg: Springer.

Cameron, C. (2014a). Developing an affirmative model of disability and impairment. In J. Swain, S. French, C. Barnes \& C. Thomas (Hrsg.), Disabling barriers - Enabling environment (3. Aufl., S. 24-30). Los Angeles: Sage.

Cameron, C. (2014b). The personal tragedy model. In C. Cameron (Hrsg.), Disability studies. A student's guide (S. 116-119). Los Angeles: Sage.

Connell, R. (2011). Southern bodies and disability: re-thinking concepts. Third World Quarterly, 32(8), 1369-1381.

Corbin, J.M.\& Strauss, A.L. (2004). Weiterleben lernen. Verlauf und Bewältigung chronischer Krankheit (2. Aufl.). Bern, Göttingen, Toronto, Seattle: Huber.

Darling, R. B. (2003). Towards a model of changing disability Identities: a proposed typology and research agenda. Disability \& Society, 18(7), 881-895.

Darling, R. B. \& Heckert, D. A. (2010). Orientations toward disability: Differences over the life course. International Journal of Disability, Development and Education, 57(2), 131-143.

Deal, M. (2003). Disabled people's attitudes towards other impairment groups: a hierarchy of impairment. Disability \& Society, 18(7), 879-910.

Dunn, D. S. \& Burcaw, S. (2013). Disability identity: Exploring narrative accounts of disability. Rehabilitation Psychology, 58(2), 148-157.

Filipp, S.-H. \& Aymanns, P. (2010). Kritische Lebensereignisse und Lebenskrisen. Vom Umgang mit den Schattenseiten des Lebens. Stuttgart: Kohlhammer.

Filipp, S.-H. \& Ferring, D. (2002). Die Transformation des Selbst in der Auseinandersetzung mit kritischen Lebensereignissen. In G. Jüttemann \& H. Thomae (Hrsg.), Persönlichkeit und Entwicklung (S. 191-221). Weinheim, Basel: Beltz Taschenbuch Verlag.

Forber-Pratt, A. J., Lyew, D. A., Mueller, C. \& Samples, L. B. (2017). Disability identity development: A systematic review of literature. Rehabilitation Psychology, 62(2), 198-207.

Forber-Pratt, A.J. \& Zape, M.P. (2017). Disability identity development model: Voices from the ADAgeneration. Disability and Health Journal, 10(2), 350-355.

French, S. \& Swain, J. (2004). Whose tragedy? Towards a personal non-tragedy view of disability. In J. Swain, S. French, C. Barnes \& C. Thomas (Hrsg.), Disabling barriers - Enabling environment (2. Aufl., S. 34-40). Los Angeles: Sage.

Gill, C. J. (1997). Four types of integration in disability identity development. Journal of Vocational Rehabilitation, 27(9), 39-46.

Goodley, D. (2013). Disability and psychology. In J. Swain, S. French, C. Barnes \& C. Thomas (Hrsg.), Disabling barriers - Enabling environment (3. Aufl., S. 61-69). Los Angeles: Sage. 
Gugutzer, R. \& Schneider, W. (2007). Der »behinderte« Körper in den Disability Studies. Eine körpersoziologische Grundlegung. In A. Waldschmidt \&W. Schneider (Hrsg.), Disability Studies, Kultursoziologie und Soziologie der Behinderung (S. 31-53). Bielefeld: transcript.

Hahn, H.D. \& Belt, T.L. (2004). Disability identity and attitudes towards cure in a sample of disabled activists. Journal of Health and Social Behavior, 45(4), 453-464.

Hildenbrand, B. (2009). Die »Bewältigung« chronischer Krankheit in der Familie - Resilienz und professionelles Handeln. In D. Schaeffer (Hrsg.), Bewältigung chronischer Krankheit im Lebenslauf (S. 137-154). Bern: Verlag Hans Huber.

Hirschberg, M. (2009). Behinderung im internationalen Diskurs. Die flexible Klassifizierung der Weltgesundheitsorganisation. Frankfurt am Main: Campus.

Hughes, B. \& Paterson, K. (1997). The social model of disability and the disappearing body. Towards a sociology of impairment. Disability \& Society, 12(3), 325-340.

Krämer, L. \& Bengel, J. (2016). Chronische körperliche Krankheit und Krankheitsbewältigung. In J. Bengel \& O. Mittag (2016), Psychologie in der medizinischen Rehabilitation. Ein Lehr-und Praxishandbuch (S. 25-36). Berlin, Heidelberg: Springer.

Knoll, N., Scholz, U. \& Rieckmann, N. (2017). Einführung Gesundheitspsychologie (4. Aufl.). Stuttgart: UTB. Lazarus, R. S. \& Folkman, S. (1984). Stress, appraisal, and coping. New York: Springer.

Lindemann, G. (2005). Die Verkörperung des Sozialen. Theoriekonstruktionen und empirische Forschungsperspektiven. In M. Schroer (Hrsg.), Soziologie des Körpers (S. 114-138). Frankfurt am Main: Suhrkamp.

Longmore, P.K. (1995). The second phase: from disability rights to disability culture. https://www. independentliving.org/docs3/longm95.html (27.03.2018).

Lude, P. \& Geyh, S. (2015). Neue Ansätze. In W. Strubreither, M. Neikes, D. Stirnimann, J. Eisenhuth, B. Schulz \&. Lude (Hrsg.), Klinische Psychologie bei Querschnittlähmung. Psychologische und psychotherapeutische Interventionen bei psychischen, somatischen und psychosozialen Folgen (S. 223-228). Wien: Springer.

Lude, P. \& Strubreither, W. (2015). Psychologische Theorien zur Bewältigung. In W. Strubreither, M. Neikes, D. Stirnimann, J. Eisenhuth, B. Schulz \& P. Lude (Hrsg.), Klinische Psychologie bei Querschnittlähmung. Psychologische und psychotherapeutische Interventionen bei psychischen, somatischen und psychosozialen Folgen (S. 183-222). Wien: Springer.

Mäder, M. (2015). Zielsetzungen in der Rehabilitation. In W. Strubreither, M. Neikes, D. Stirnimann, J. Eisenhuth, B. Schulz \& P. Lude (Hrsg.), Klinische Psychologie bei Querschnittlähmung. Psychologische und psychotherapeutische Interventionen bei psychischen, somatischen und psychosozialen Folgen (S. 15-20). Wien: Springer.

Mürner, C. \& Sierck, U. (Hrsg.). (2011). Behinderte Identität? Neu-Ulm: AG-SPAK.

Nario-Redmond, M. R., Noel, J.G. \& Fern, E. (2013). Redefining disability, re-imagining the self: Disability identification predicts self-esteem and strategic response to stigma. Self and Identity, 12(5), 468-488.

Nario-Redmond, M. R. \& Oleson, K. C. (2016). Disability group identification and disability-rights advocacy: Contingencies among emerging and other adults. Emerging Adulthood, 4(3), 207-218.

Oliver, M. \& Barnes, C. (2012). The new politics of disablement. London: Palgrave.

Olkin, R. (2003). Can disability studies and psychology join hands? American Psychologist, 58(4), 296-304.

Olkin, R. (2012). Disability: A primer for therapist. In E. M. Altmaier \& J.-I.C. Hansen (Hrsg.), The Oxford handbook of counseling psychology (S. 460-479). New York: Oxford University Press.

Putnam, M. (2005). Conceptualizing disability. Development a framework for political disability identity. Journal of Disability Policy Studies, 16(3), 188-198.

Reeve, D. (2006). Towards a psychology of disability. The emotional effects in a disabling society. In D. Goodley \& R. Lawthom (Hrsg.), Disability and psychology. Critical introductions and reflections (S. 94-107). London: Palgrave. 
Sherry, M. (2016). A sociology of impairment. Disability \& Society, 31(6), 729-744.

Swain, J. \& French, S. (2000). Towards an affirmation model of disability. Disability \& Society, 15(4), $569-582$.

Tajfel, H. \& Turner, J.C. (1979). An integrative theory of intergroup conflict. In W. G. Austin \& S. Worchel (Hrsg.), The social psychology of intergroup relations (S. 33-47). Monterey: Brooks/Cole.

Thomas, C. (1999). Female forms: Experiencing and understanding disability. Buckingham, Philadelphia: Open University Press.

Tremain, S. (2002). On the subject of impairment. In M. Corker \& T. Shakespeare (Hrsg.), Disability/postmodernity. Embodying disability theory (S. 32-47). London, New York: continuum.

Union of the Physically Impaired Against Segregation (UPIAS) (1976). Fundamental principles of disability. London: UPIAS.

Waldschmidt, A. (2005). Disability Studies: Individuelles, soziales und/oder kulturelles Modell von Behinderung? Psychologie und Gesellschaftskritik, 29(1), 9-31.

Wehmeyer, M. L. (Hrsg.). (2013). The Oxford handbook of positive psychology and disability. New York: Oxford University Press.

Wendell, S. (1999). Feminismus, Behinderung und die Transzendenz des Körpers. Deutsche Zeitschrift für Philosophie, 47(5), 803-815.

Wenzel, T.-R. \& Morfeld, M. (2017). Nutzung der ICF in der medizinischen Rehabilitation in Deutschland: Anspruch und Wirklichkeit. Bundesgesundheitsblatt - Gesundheitsforschung - Gesundheitsschutz, 60(4), 386-393.

Wolf-Kühn, N. \& Morfeld, M. (2016). Rehabilitationspsychologie. Wiesbaden: Springer.

Zander, M. (2015). Chronische Krankheiten aus der Perspektive der Disability Studies. Sozialmagazin, 40(7/8), 32-39.

\section{Die Autorin}

Birgit Behrisch, Dr. phil., ist Gastprofessorin für inklusive Pädagogik an der Katholischen Hochschule für Sozialwesen Berlin mit den Arbeitsschwerpunkten Inklusion und Teilhabe, Partizipative Forschung und Wissenssoziologie.

Kontakt:Köpenicker Allee39-57,10318Berlin,E-Mail:birgit.behrisch@khsb-berlin.de 